electric field containing a preformed $\mathrm{pH}$ gradient. The antigen was found to focus in two discrete zones corresponding to two isoelectric points. The zones in which focusing occurred were at pH 3.95 and 4.90 for the ay subtype of hepatitis $B$ antigen and pH 3.62 and 4.33 for the ad subtype. Both zones were free of serum proteins and were found to contain intact $22 \mathrm{~nm}$ spherical particles characteristic of this antigen when examined by immune electron microscopy. The results reveal a difference between the two major subtypes in the $\mathrm{pH}$ values at which electrofocusing occurs, although one of these zones may contain material common to both subtypes. Further studies on the chemical multiformity of hepatitis $B$ antigen may enhance the detection potential of available serological tests for the antigen, including the preparation of purified reagents for radioimmunoassay.

Radioimmunoassay, which combines the specificity of antigen-antibody interaction with the sensitivity of radioisotope detection, is the most sensitive technique currently available for detecting hepatitis $B$ antigen. Comparative tests have shown that the sensitivity of radioimmunoassay is 2000 to 10000 times greater than immunodiffusion for detection of antigen and 10000 to 1000000 times more sensitive than immunodiffusion for detection of antibody. The specificity of the technique was confirmed by concentrating serum which was positive only by radioimmunoassay and examination by electron microscopy after negative staining.

Human Lymphocyte Antigens as a Possible Diagnostic Aid in Ankylosing Spondylitis

M. CAFFREY, D. A. BREWERTON, F. D. HART, AND D. C. O. JAMES (Blood Transfusion Unit, Westminster Hospital, London) To aid the diagnosis of ankylosing spondylitis in patients a study of a genetic parameter was made.

Human lymphocyte antigens were determined for 85 unrelated ankylosing spondylitic patients using a standard lymphocytotoxicity test. Twenty-six human lymphocyte antigens were studied and significant differences were observed between the frequency of the HL-A 27 in ankylosing spondylitic patients $(95 \%)$ and normal controls in a Caucasian population $(5 \%)$.

The finding that the antigen HL-A 27 appears to have a higher frequency among certain isolated communities, where there is a greater incidence of ankylosing spondylitis than in others, lends support to the above observation. Likewise, its low frequency among negroid populations, which have a very low incidence of this disease, is also of interest.

The presence of HL-A 27 among these patients with such a high frequency suggests that the observation could be used as a diagnostic aid.

\section{Some Interesting Serological Findings in Patients with Epilepsy}

P. HAMILton (City Hospital, Aberdeen; introduced by R. J. L. DAVIDSON) The (chance observation in our laboratory that apparently false positive screening tests for infectious mononucleosis (IM) could occur in epileptic patients prompted us to undertake this study of 119 epileptic and 62 non-epileptic control patients from the same long-stay hospital. Investigations included peripheral blood examination, immunoglobulin analysis, direct Coombs, LE-latex, and three commercially available IM slide tests. Differential absorption and EB virus antibody tests were performed on selected groups.

Positive IM slide tests were obtained in $35(29 \%)$ of the epileptic patients compared with seven $(11 \%)$ in the control group. None of these positively reacting patients had a positive differential absorption test nor a white cell pattern diagnostic of infectious mononucleosis. Further, their age distribution was not typical of the clinically manifest disease. The multiplicity of anticonvulsant drug regimens made analysis difficult but significantly about a third of patients receiving phenobarbitone and/or primidone had positive IM slide reactions. Twenty-eight of $35(80 \%)$ IM-positive epileptic patients had raised IgG and/or IgM levels compared with 45 of $84(53 \%)$ IM-negative epileptic patients. Very low IgA levels were found in $31(26 \%)$ of the epileptic patients. The incidence of positive LE-latex tests was similar in both epileptic and non-epileptic groups: of the 18 positively reacting sera 16 had raised IgM levels. EB virus antibody titres were generally higher in those patients with positive rather than negative IM tests.

The importance of recognizing the high incidence of false positive IM slide reactions in patients receiving anticonvulsants is emphasized. Some of the implications of the overall findings, including their possible relevance to an immunosuppressive effect of anticonvulsant drugs, are briefly discussed.

An Assessment of Acid and Alkaline Phosphatase Determinations in the Diagnosis of Prostatic Cancer

D. M. GOLDBERG AND G. Ellis (The Royal Hospital, Sheffield) Serum acid phosphatase (AcPase) was routinely measured by the method of Ellis, Belfield, and Goldberg (1971) on 389 patients grouped as follows: (a) untreated carcinoma of prostate (45 cases); (b) treated carcinoma of prostate (22 cases); (c) benign prostatic hypertrophy-biopsy positive 
( 52 cases); (d) benign prostatic hypertrophy-clinical diagnosis only (60 cases); (e) other cancers (57 cases); (f) other urological illness (22 cases); (g) miscellaneous disease (116 cases); (h) undiagnosed (15 cases). The mean activity (SD) of the miscellaneous group was $1.97(1.07) \mathrm{IU} /$ litre at $37^{\circ} \mathrm{C}$, giving an upper normal limit (mean +2 SD) of $4 \cdot 1$ IU/litre. The AcPase values in this group did not correlate with age $(r=0.040)$. Eight patients with untreated carcinoma of prostate $(22 \%)$ had values $\leqslant 4 \cdot 1 \mathrm{IU} /$ litre. Six of these had no prostatic enlargement and no metastases; the remaining two had enlarged prostates but no evidence of metastases. All 28 patients in this series with soft tissue (7) or bony (21) metastases had AcPase $>4 \cdot 1 \mathrm{IU} /$ litre. Values $>4.1 \mathrm{IU} /$ litre were found in seven cases $(6 \%)$ of benign hypertrophy, three cases $(5 \%)$ of non-prostatic cancer, two cases $(2 \%)$ of miscellaneous illness, and one case $(6 \%)$ of uncertain diagnosis. No values $>4 \cdot 1 \mathrm{IU} /$ litre were found in the patients with other urological illness.

Sixty per cent of patients ( 26 cases) with untreated prostatic carcinoma had elevated serum alkaline phosphatase activity (APase), ie, $>15 \mathrm{KAU} / 100 \mathrm{ml}$ as determined by an AutoAnalyzer adaptation of the method of Kind and King (1954). Correlation between the two phosphatases was not significant $(1=0.294 ; \mathbf{P}>0.05)$. APase was elevated in the presence of normal AcPase in only three cases of prostatic cancer, but all three were without metastases. Two further cases with minimal elevation of AcPase had APase $>50 \mathrm{KAU} / 100 \mathrm{ml}$. While APase is less frequently elevated than AcPase in prostatic cancer patients, it provides valuable confirmation of the diagnosis when the latter is normal or equivocal.

\section{References}

Ellis, G., Belfield, A., and Goldberg, D. M. (1971). J. clin. Path., 24. 493.

Kind, P. R. N., and King, E. J. (1954). J. clin. Path., 7, 322.

\section{A Survey of Hepatitis in Laboratories}

N. R. GRIST (Department of Infectious Diseases, University of Glasgow) A preliminary enquiry from ACP members suggested a rising incidence of hepatitis which was reported from 58 of 285 laboratories for 1964-69 from 32 of 289 for 1970. Of 127 cases, 87 involved technicians and 48 affected workers in biochemistry. A continuing more detailed enquiry was initiated with the following results:

Information for 1970 from 244 laboratories showed 13 hepatitis cases in 12 laboratories. Attack rates were highest in science microbiologists $(1 / 126=$ $0.79 \%)$ and biochemistry technicians $(7 / 1438=$ $0.49 \%)$, the overall rate being $13 / 11253(0 \cdot 116 \%)$.
Information for 1971 from 215 laboratories showed 18 hepatitis cases in 15 laboratories. Attack rates were highest in science biochemists $(2 / 315=$ $0.63 \%)$, medical haematologists $(2 / 315=0.64 \%)$, and medical morbid anatomists $(2 / 390=0.51 \%)$; no cases were reported among biochemistry technicians.

Hepatitis B ('Australia') antigen was found in 11 of 22 cases tested over the two years and was associated with less mild illness; there was no death.

Among laboratories with hepatitis cases, more than average tested specimens from haemodialysis, transplant, and haemophilia units, and from drug addiction centres (1971 but not 1970). Risk was unaffected whether high-risk specimens were tested by general staff, special unit staff, or not accepted, or whether they were tested on mechanized apparatus in general use or segregated or not tested on such apparatus. Only slightly increased risk was found in laboratories testing for Australia antigen.

\section{Bacteraemia in the Burned Patient}

H. J. K. M. MAMATTAH, C. H. BEARD, AND D. M. JONES (Withington Hospital, Manchester) Burns are regularly colonized by a variety of bacteria, often without clinical evidence of infection. In some patients, due to various factors, colonization progresses to invasion and septicaemia. A study has been made of the influence of operative procedures, ie, desloughing and excision of necrotic tissue on the infective process. A series of unselected operations were monitored by taking a series of blood cultures while the operations were in progress. Out of 52 blood cultures, $17(33 \%)$ were positive and nearly half of these grew more than one organism. Bacteraemia was detected in $40 \%$ of the operations studied and often appeared to be of 10 to 20 minutes duration. The bacterial species grown by blood culture were always present colonizing the burn although not all the colonizing species were recovered in the blood cultures. Attempts were made to relate the findings to the severity of the burn, to the magnitude of the operative procedure, and to the time that had elapsed between the burn and the operation. In spite of bacteraemia none of the patients studied were treated with antibiotics and in no case was systemic infection established. Immunoglobulin levels are depressed for a few days after the patient is burnt but in the patients studied immunoglobulin levels were found to be normal at the time of operation and bacteraemia. The average timing of the operations was 20 to 25 days after the burn. Comparison of these results with observations made when septicaemia became established following 